\title{
Identification of Functional Regulatory Residues of the $\beta$-Lactam Inducible Penicillin Binding Protein in Methicillin- Resistant Staphylococcus aureus
}

\author{
Andreas N. Mbah and Raphael D. Isokpehi \\ Center for Bioinformatics \& Computational Biology, Department of Biology, Jackson State University, Jackson, MS 39217, USA \\ Correspondence should be addressed to Andreas N. Mbah; nji41@yahoo.com
}

Received 27 April 2013; Revised 19 June 2013; Accepted 3 July 2013

Academic Editor: Spyros Pournaras

Copyright (c) 2013 A. N. Mbah and R. D. Isokpehi. This is an open access article distributed under the Creative Commons Attribution License, which permits unrestricted use, distribution, and reproduction in any medium, provided the original work is properly cited.

\begin{abstract}
Resistance to methicillin by Staphylococcus aureus is a persistent clinical problem worldwide. A mechanism for resistance has been proposed in which methicillin resistant Staphylococcus aureus (MRSA) isolates acquired a new protein called $\beta$-lactam inducible penicillin binding protein (PBP-2'). The PBP-2' functions by substituting other penicillin binding proteins which have been inhibited by $\beta$-lactam antibiotics. Presently, there is no structural and regulatory information on PBP- $2^{\prime}$ protein. We conducted a complete structural and functional regulatory analysis of PBP-2' protein. Our analysis revealed that the PBP-2' is very stable with more hydrophilic amino acids expressing antigenic sites. PBP- $2^{\prime}$ has three striking regulatory points constituted by first penicillin binding site at Ser25, second penicillin binding site at Ser405, and finally a single metallic ligand binding site at Glu657 which binds to $\mathrm{Zn}^{2+}$ ions. This report highlights structural features of PBP-2' that can serve as targets for developing new chemotherapeutic agents and conducting site direct mutagenesis experiments.
\end{abstract}

\section{Introduction}

Methicillin resistance (MR) by Staphylococcus aureus is a persistent clinical problem affecting many geographic locations worldwide [1-4]. Glycopeptides such as vancomycin and teicoplanin are often the choice in treating infections associated with methicillin-resistant $S$. aureus (MRSA), at times with little success [5]. The resistance of methicillin by Staphylococcus aureus has been documented to depend on several factors such as temperature [6], $\mathrm{pH}$ [7], $\mathrm{NaCl}$ concentration, and inoculum size $[6,8]$. Even though methicillinresistant staphylococci produce penicillinase, blocking of this enzyme do not affect the level of methicillin resistance [9]. The history of ever-increasing resistance among MRSA strains suggests that they are likely to be more prevalent in the future, thus severely restricting treatment options [10].

Penicillin-binding proteins (PBPs) are enzymes commonly expressed by MRSA during peptidoglycan synthesis, cell growth, and morphogenesis. The PBPs are inhibited by
$\beta$-Lactam antibiotics such as methicillin and vancomycin by interrupting the biochemical functions at the D-Ala-DAla terminus of the peptidoglycan precursor $[5,11]$. The expressions of PBPs in MRSA have been well documented in previous studies, with $\mathrm{PBP}-2$ and $\mathrm{PBP}-3$ proposed as the lethal targets for $\beta$-lactams action [12-14]. However, another mechanism for methicillin resistance have been reported in which MRSA isolates have acquired a new PBP protein called $\beta$-lactam inducible penicillin binding protein (PBP2a or PBP$2^{\prime}$ ) [1]. The induction of PBP-2' occurs only in the presence of penicillinase plasmid, and PBP-2' can be produced constitutively in MRSA which had lost the penicillinase plasmid $[1,15]$. The PBP- $2^{\prime}$ protein has low affinity for penicillin and most other $\beta$-lactam antibiotics. The PBP- $2^{\prime}$ is capable of substituting for other PBPs during cell wall synthesis after $\beta$ lactams antibiotics have inhibited them [16]. In this model, PBP- $2^{\prime}$ acts as a surrogate enzyme capable of taking over the normal functions of staphylococcal PBPs in cell wall biosynthesis. The ability of PBP-2 ${ }^{\prime}$ to affect cell wall synthesis 
in the presence of methicillin requires cooperation from the transglycosylase domain of the native PBP-2 $[17,18]$.

The amino acid sequence of PBP- $2^{\prime}$ is similar to those of the shape-determining protein (PBP-2) and septum-forming (PBP3) of Escherichia coli [10]. This support the idea that PBP-2' might have evolved as a combination of two genes of inducible type I penicillinase gene and a PBP gene which coordinate the expression of beta-lactam-inducible MRSA PBP [19]. The PBP-2' protein is encoded by the mecA gene carried on a large mobile genetic element also known as SCCmec [20-22], which integrates it into the chromosome of MRSA strains. However, MRSA PBP-2' expression is modulated by a transacting factor in response to the presence of the cell wall-active antibiotics such as methicillin, vancomycin, and oxacillin [10]. Thus, PBP- $2^{\prime}$ is proposed as a vital contributor in the increased prevalence of MSRA $[1,17,18]$.

Presently few therapeutic alternatives exist for the treatment of MRSA infections. This research report presents structural features that regulate the functioning of PBP$2^{\prime}$. These features could be exploited in developing new chemotherapies against MRSA. Our analysis reveals that the PBP- $2^{\prime}$ protein has three striking regulatory points constituted by first penicillin binding site at Ser 25 , second penicillin binding site at Ser405 and finally a single metallic ligand binding site at Glu657 which binds to $\mathrm{Zn}^{2+}$ ions. These structural features can be exploited as novel targets for developing new drugs against MSRA.

\section{Materials and Method}

2.1. Sequence Retrieval, Amino Acid, and Physicochemical Parameters Analysis. The beta-lactam-inducible penicillin binding protein (PBP-2') (UniProt ID: P07944 | PBP STAAU) reviewed sequence was retrieved from UniProt protein database (UniProt release 2011_11) (http://www .uniprot.org/). The amino acid composition of the sequence was computed using the ProtParam tool (http://www.expasy .ch/cgi-bin/protparam). The ProtParam tool was also used to compute the physicochemical parameters such as theoretical isoelectric point (Ip), molecular weight, total number of positive and negative residues, extinction coefficient, halflife, instability index, aliphatic index, and grand average hydropathy (GRAVY). The percentages of hydrophobic and hydrophilic residues were calculated from the primary structure analysis and the hydrophobicity plot was done using both Hopp-Woods and Kyte-Doolottle scale for possible antigenicity.

2.2. Conserved Domain Search, Homology Modeling, and Visualization of $3 D$ Structure. The possible conserved domains regulating the functional mechanism of $\mathrm{PBP}-2^{\prime}$ protein were analyzed using the NCBI public server at http:// www.ncbi.nlm.nih.gov/Structure/cdd/wrpsb.cgi, UniProt pr otein database (UniProt release 2011_11) (http://www.uniprot.org/), and Pfam database at http://pfam.sanger.ac.uk/ search/. Their functional units and domain residues were identified and documented. The three-dimensional (3D) structure of (PBP-2') encoded protein was modeled using the PDB template 1mwu (Chain A). The
TABle 1: Amino acid composition of PBP-2' computed using ProtParam server.

\begin{tabular}{lccc}
\hline $\begin{array}{l}\text { Amino } \\
\text { acid }^{*}\end{array}$ & Composition (\%) & Hydrophilic (\%) & Hydrophobic (\%) \\
\hline Ala & 3.9 & 2.1 & 3.9 \\
Arg & 2.1 & 8.5 & \\
Asn & 8.5 & 7.5 & \\
Asp & 7.5 & & \\
Cys & 0.0 & 3.4 & \\
Gln & 3.4 & 6.0 & \\
Glu & 6.0 & & 9.0 \\
Gly & 7.0 & 1.6 & 6.9 \\
His & 1.6 & & \\
Ile & 9.3 & & \\
Leu & 6.9 & 13.6 & \\
Lys & 13.6 & & \\
Met & 2.7 & & \\
Phe & 2.4 & & \\
Pro & 2.5 & 6.5 & \\
Ser & 6.3 & 4.3 & \\
Thr & 4.3 & & \\
Trp & 1.0 & & \\
Tyr & 5.5 & & \\
Val & 5.5 & & \\
\hline Total & 100.0 & & \\
\hline & 0.3 & \\
\hline
\end{tabular}

${ }^{*}$ The composition of each amino acid residue is indicated in percentage. The composition of hydrophilic amino acids is $53.3 \%$ while hydrophobic amino acids constitute $35.1 \%$. The protein can be described as moderately hydrophilic.

three-dimensional (3D) structure of the gene was determined using the following servers; SwissModel server (http://www.swissmodel.expasy.org/) and the Phyre/Phyre2 server (http://www.sbg.bio.ic.ac.uk/phyre2/html/page.cgi?id =news) [23]. The quality of the model was evaluated with Ramachandran plot data, based on the phi-psi torsion angles of all the residues in the model using DeepView-Swiss-PdbViewer (http://spdbv.vital-it.ch/). The Ramachandran plot obtained from DeepView was further assessed using Ramachandran plot 2 assessment server (http://dicsoft1.physics.iisc.ernet.in/rp/). The Rasmol tool (http://www.openrasmol.org/) was used in visualizing the modeled 3D structures and the distribution of the secondary structures. The three-dimensional (3D) LigandSite residues and the predicted ligand of the PBP- $2^{\prime}$ protein were determined using 3DLigandSite server at (http://www.sbg.bio.ic.ac.uk/3dligandsite/) [24]. The Rasmol tool was further employed in locating the positions of the ligand binding sites and the ligand on the 3D structure with particular attention to the regulatory points for PBP- $2^{\prime}$.

\section{Results and Discussion}

3.1. Amino Acid Content and Physicochemical Parameters. The analysis suggests that this protein is hydrophilic due 
TABLe 2: Physicochemical properties of PBP-2' computed using ProtParam server.

\begin{tabular}{lc}
\hline ProtParam parameters & Values \\
\hline No. of amino acids & 670 \\
Molecular weight & $76463.2 \mathrm{Da}$ \\
Theoretical pI & 9.09 \\
No. of negative charge residues & 90 \\
No. of positive charge residues & 105 \\
Formula & $\mathrm{C}_{3415} \mathrm{H}_{5428} \mathrm{~N}_{912} \mathrm{O}_{1039} \mathrm{~S}_{18}$ \\
Extinction coefficient & $93630 \mathrm{M}^{-1} \mathrm{~cm}^{-1}$ \\
Estimated half-life & 30 hours $^{\circ}$ \\
Instability index & 30.08 \\
Aliphatic index & 82.76 \\
Grand average of hydropathicity (GRAVY) & -0.698 \\
Total number of atoms & 10812 \\
\hline
\end{tabular}

${ }^{*}$ The physicochemical parameters define the protein chemical and physical properties in its native state. The protein has a net positive charge and is basic in nature $(\mathrm{pI}>7)$.

to the presence of high polar amino acid residues (53.3\%) against nonpolar (hydrophobic) amino acids residues (35.1\%) (Table 1). The protein can be described as moderately hydrophilic. The hydrophobic residues are usually found in the core of most proteins, and they help in stabilizing the proteins through the numerous van der Waal interactions [25]. The hydrophilic residues are located mostly at the surface active sites of proteins, where they interact with other polar residues or with water molecule. The PBP-2' protein is made of 670 amino acid residues with an average molecular weight of $76463.2 \mathrm{Da}$. The analysis indicates that there are more Lys, Ile, Asn, Asp, Gly, and Ser in that order (Table 1). The atomic composition (10812 atoms) consists of 3415 carbons $(\mathrm{C}), 5428$ hydrogen $(\mathrm{H}), 912$ nitrogen $(\mathrm{N}), 1039$ oxygen $(\mathrm{O})$, and 18 sulfur atoms with a molecular formula of $\mathrm{C}_{3415} \mathrm{H}_{5428} \mathrm{~N}_{912} \mathrm{O}_{1039} \mathrm{~S}_{18}$ (Table 2). The 18 sulfur atoms were constituted by methionine residues present in the primary structure. The computed pI (9.09) [pI > 7] indicates that the protein is basic in nature. The isoelectric point (pI) indicates the $\mathrm{pH}$ at which the protein surface is covered with charge [26], and the net charge of this protein is positive. The high number of positively charged residues (Arg + Lys $=105)$ against the total number of negatively charged residues (Asp $+\mathrm{Glu}=90)$ is the main contributing factor to the positive charge. At a given pI, proteins are stable and compact; thus, this parameter will be useful for developing buffer systems for purification of this protein by isoelectric focusing techniques [27].

The ProtParam extinction coefficient at a wavelength of $280 \mathrm{~nm}$ measured in water is favorable because proteins are able to absorb strongly at this wavelength than other substances that may be commonly found in the solution. The extinction coefficient for this protein was computed with respect to Trp and Tyr present in the primary structure. The extinction coefficient of $93630 \mathrm{M}^{-1} \mathrm{~cm}^{-1}$ at $280 \mathrm{~nm}$ wavelength computed for this protein was constituted by individual contributions from $\operatorname{Trp}(1.0 \%)$ and Tyr (5.5\%), respectively. This suggests that $\mathrm{PBP}-2^{\prime}$ protein can be analyzed using UV spectrum assay [28]. The computed protein concentration and the extinction coefficient will contribute immensely in the quantitative analysis of the protein-protein and protein-ligand interaction of this protein in solutions $[28,29]$. The estimated half-life of this protein with Met as the N-terminal of the sequence was $30(>20)$ hours. The concentrations of Ala (3.9\%), Leu (6.9\%), and Val (5.5\%) may be contributing to the stability of the protein [30,31]. The halflife of these 3 mentioned residues had been well documented in the mammalian with values of Ala (4.4 hours), Leu (5.5 hours), and Val (100 hour). In other organisms, these residues are also contributing to the protein stability. In yeast, the halflife was the same ( $>20$ hours) for Ala and Val, and both were the same in $E$ coli with values $>10$ [32].

Based on the predicted instability index, the ProtParam tool indicates that the protein is stable with a value of 30.08. This parameter was computed based on the impact of dipeptides in the protein sequence [33]. This protein is shown to have high aliphatic index, half-life, and large amount of hydrogen atoms (5428) to form strong hydrogen bonds. Such hydrogen bonds are known to impact significant stability to proteins, making them resistance to degradation [26]. Therefore the formation of hydrogen bonds is contributing to its stability. The stability value is a measure of the protein stability in a test tube [26]. A protein of instability index $<40$ is considered as stable, while those with values $>40$ are unstable [33].

The aliphatic index (AI) of a protein is the relative volume occupied by the aliphatic side chains (alanine, valine, isoleucine and leucine) and is taken as contributor to the increase thermal stability of globular proteins. The aliphatic index computed for this protein was 82.76 using a formulated rule illustrated by [34]. This high aliphatic index indicates that the protein can be stable within a wide range of temperature. Proteins with low thermal stability turn to be more structurally flexible. The grand average of hydropathy (GRAVY) is the computed sum of hydropathy values of all the amino acids, divided by the number of residues in the sequence [35]. The very low GRAVY index $(-0.698)$ of this protein indicates that the protein is very reactive in water, which might be a contributing factor to MRSA virulence and pathogenicity. The Hopp-Woods scale identified three regions on this polypeptide predicted to be highly hydrophilic. These hydrophilic regions are exposed on the surface and may possibly be antigenic [36]. They are shown to have peak values greater than 0 (Figure 1). This indicates that this protein can be served as a possible vaccine target.

3.2. Conserved Domain and Functional Regulatory Point Analysis of MRSA PBP-2' Gene. Methicillin-resistant Staphylococcus aureus is a leading cause of hospital carried infections worldwide and is a leading community-associated pathogen [37]. These Staphylococcus strains are cross-resistant to virtually all $\beta$-lactam antibiotics clinically used worldwide [38]. The MRSA clinical strains are often multidrug resistant, thus limiting the therapeutic options for staphylococcal infections [39]. The conserved domains in proteins are responsible 


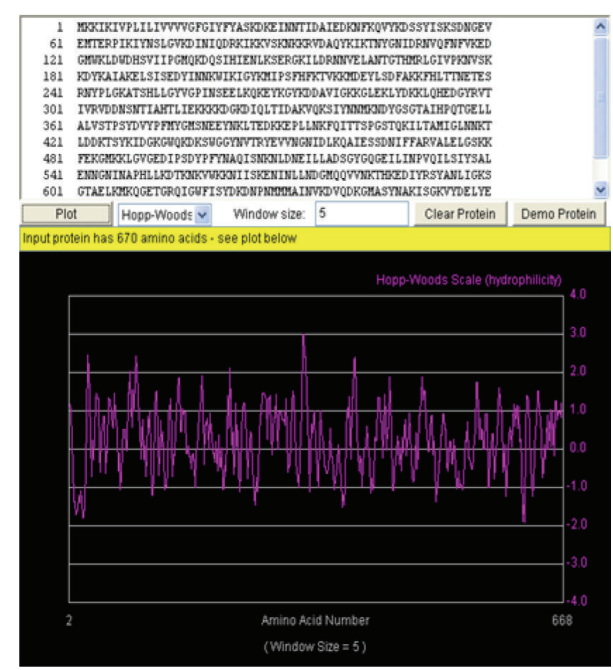

(a)

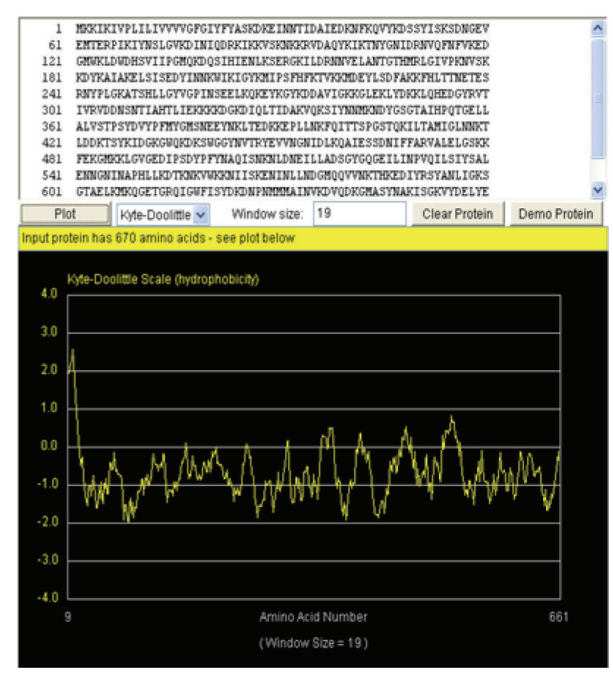

(b)

Figure 1: The hydropathy plot for PBP-2' protein. The yellow plot (b) is the Kyte-Doolittle hydrophobicity plot. Sections of the plot with high values $>0.0$ are highly hydrophobic or membrane spanning segments. The magenta plot (a) is the Hopp-Wood hydrophilicity plot. Higher values above $>0.0$ predict rich charge exposed regions with potential antigenic site. PBP2 ${ }^{\prime}$ gene shows potential antigenic sites with values $\geq 2$. Above the plots are the $\mathrm{PBP} 2^{\prime}$ amino acids sequence with 670 residues.

for many important biological functions within the cellular process of an organism [40-43]. The conserved domain search for PBP-2 $2^{\prime}$ protein revealed 3 functional distinct domains within its amino acids sequence. These domains are MecA_N (residue 24-140), PBP-dimer (residue 146-315), and Transpeptidase (residue 347-660) (Figure 2).

The crystal structure of PBP-2' gene has not yet been determined here we present the $3 \mathrm{D}$ modeled structure of the protein using 1 mwu (Chain A) as template with $94 \%$ of the residues modeled at $>90 \%$ confidence level. The Rasmol tool was used for visualizing the tertiary structure of the PBP- $2^{\prime}$ protein. The secondary structure components composed of 31 helices, 41 sheets, 60 turns, and 5478 hydrogen bonds shown with dotted line. The MecA_N domain spans residue position 24-140, and it is depicted red in color (Figures 3 and 4) and responsible for expressing the mecA gene. The phenotypical characteristics of MRSA are due mainly to the presence of $m e c A$, which encodes a penicillin-binding protein (PBP$\left.2^{\prime}\right)$ with reduced affinity for $\beta$-lactams $[13,19]$. The genetic determinant of PBP- $2^{\prime}$, the mecA gene, is not native to $S$. aureus but was reported to be imported from an unidentified extraspecies source [44]. The mecA gene is localized in a large heterologous chromosomal cassette, the SCCmec element [45], and some MRSA strains carry upstream to the mecA gene the regulatory genes mecI-mecR1 encoding for a repressor and a sensor/inducer of the mecA expression [46]. Because mecI-mecR1 induction of mecA expression is not fast enough, functional mecI and mecR1 genes render the cell phenotypically susceptible in the presence of mecA $[46,47]$. In vitro, the deletion of the mecI gene increases the resistance levels to $\beta$-lactams in staphylococci [4648]. This suggests that complete resistance to $\beta$-lactams by MRSA might be contributed by nonfunctional mecI-mecR 1 regulatory system $[46,47]$. There are documented evidences of the accumulation of point mutations in the mecI coding sequence in several MRSA strains [49-53].

However, there is no clear relationship between the amounts of mecA transcript or $\mathrm{PBP} 2^{\prime}$ protein and the phenotypic level of resistance $[54,55]$. MRSA strains are also classified based on the type of SCCmec element they carry because the same lineage may be associated with several SCCmec types [56, 57]. Also MRSA strains are known to show strainto-strain variation in resistance level, genetic backgrounds and in their SCCmec structures that carries the resistance mecA gene [58]. In S. aureus, three major mec classes have been described based on the presence of insertion sequences and intact or disrupted mecI-mecR1 sequences. The class A has intact sequences for mecI-mecR1 whereas classes B and $\mathrm{C}$ have no mecI and have partially deleted mecRl due to the integration of insertion sequences in the regulatory region of mecA gene. Based on the SCCmec types, the mec gene complexes are grouped into three classes as follows: class A (SCCmec types II, III, and VIII); class B (SCCmec types I, IV, and VI), and class C (SCCmec types V, and VII) [39]. Similarly the epidemicity of MRSA strains does not properly correlate with the mecI-mecR1 functionality as well. This has been reported with the nosocomial MRSA clones from the ST5-II and the ST239-III which were characterized to SCCmec types II and III, respectively, even though they have a complete mecI-mecR1 locus [56].

The mecA domain also contains one of the penicillin binding sites located on amino acid residue Ser25 (color cyan) which is considered as one of the active sites of PBP-2' (Figures 3 and 4). It is located on the loop of the secondary structure and on the surface of the PBP-2' folded structure. This suggests that this site might be very unstable for penicillin binding $[59,60]$. Boronic acid bounds and inhibits the nucleophilic serine of the active site and thus 


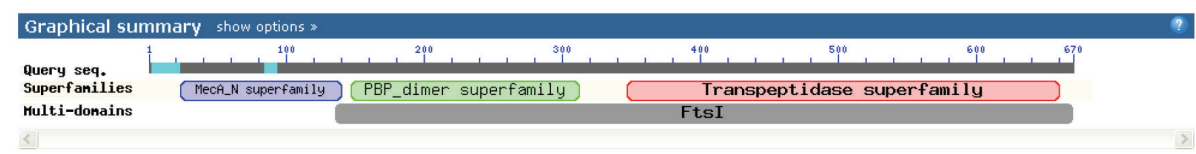

(a)

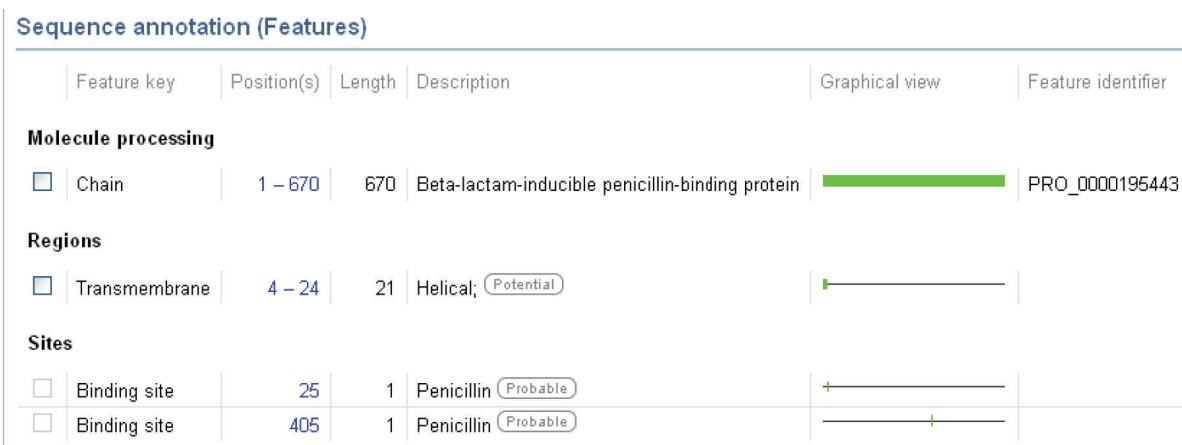

(b)

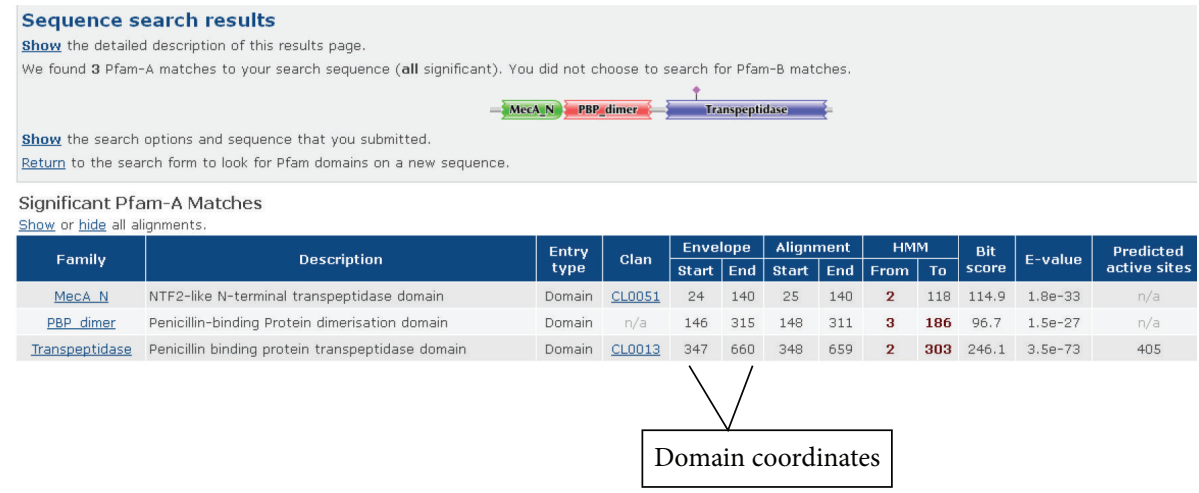

(c)

FIgURE 2: Visualization of the functional domains of PBP-2' protein using NCBI, UniProt, and Pfam domain tools. The position and span of each domain unit across the protein are shown. The span of residues contributing to the function of each domain is shown including the regulatory points. (a) is the functional domain from NCBI while (b) is the Uniprot database annotation. (c) shows the domain verification with Pfam annotation.

mimicking the transition state of the enzymatic reaction involved in the catalytic mechanism of penicillin-binding proteins [61]. The Ser25 is one of the key residues involved in the catalytic mechanism of penicillin-binding proteins, suggesting that the PBP-2' function might be regulated at this amino acid residue using amino acid substitutions, combined with site-directed mutagenesis studies [62].

The second domain is the PBP dimer which is constituted by amino acid residues 146-315 and is represented in blue (Figures 3 and 4). There is evidence suggesting that PBPs exist in dimeric forms [63]. The PBPs anchor to cytoplasmic membranes by an amino-terminal transmembrane segment $[64,65]$, and the dimer formed is less soluble than monomeric PBPs [63]. This suggests that the PBP-2' dimeric form is more tightly associated with the peptidoglycan layer and/or the outer membrane than the monomeric form. The dimeric PBPs are suggested to be located both in the outer and inner membrane fractions [66]. This indicates that the fraction of PBP-2' not associated with the cytoplasmic membrane could be the active enzyme. The dimeric form of PBP is not linked by disulfide cross-links [67]. This is in agreement with our analysis on the amino acid content of PBP- $2^{\prime}$ which lacks cysteine residues in its amino acid sequence length (Table 1). Nevertheless, these dimers involve strong noncovalent interactions [67]. There is no clear documentation on the physiological role regarding dimerization of PBP, and it remains speculative. Irrespective of this, there is suggestion that the existence of the dimer is a well monitored and carefully regulated event and recent models of peptidoglycan synthesis imply that PBP acts as a dimer within a multienzymatic complex $[68,69]$.

The third domain is the transpeptidase domain constituted by amino acid residue 347-660 (color green) (Figures 3 and 4). Several lines of evidence indicate that transpeptidase activity is the essential function of PBP2 compensated by PBP-2'. This supports the genetic evidence that PBP- $2^{\prime}$ has transpeptidase activity (TPase activity) [70]. Formerly the TPase activity of PBP-2' ${ }^{\prime}$ was assumed based on homology with other PBPs and models of methicillin resistance in S. aureus [19]. The PBP- $2^{\prime}$ is currently classified as class 


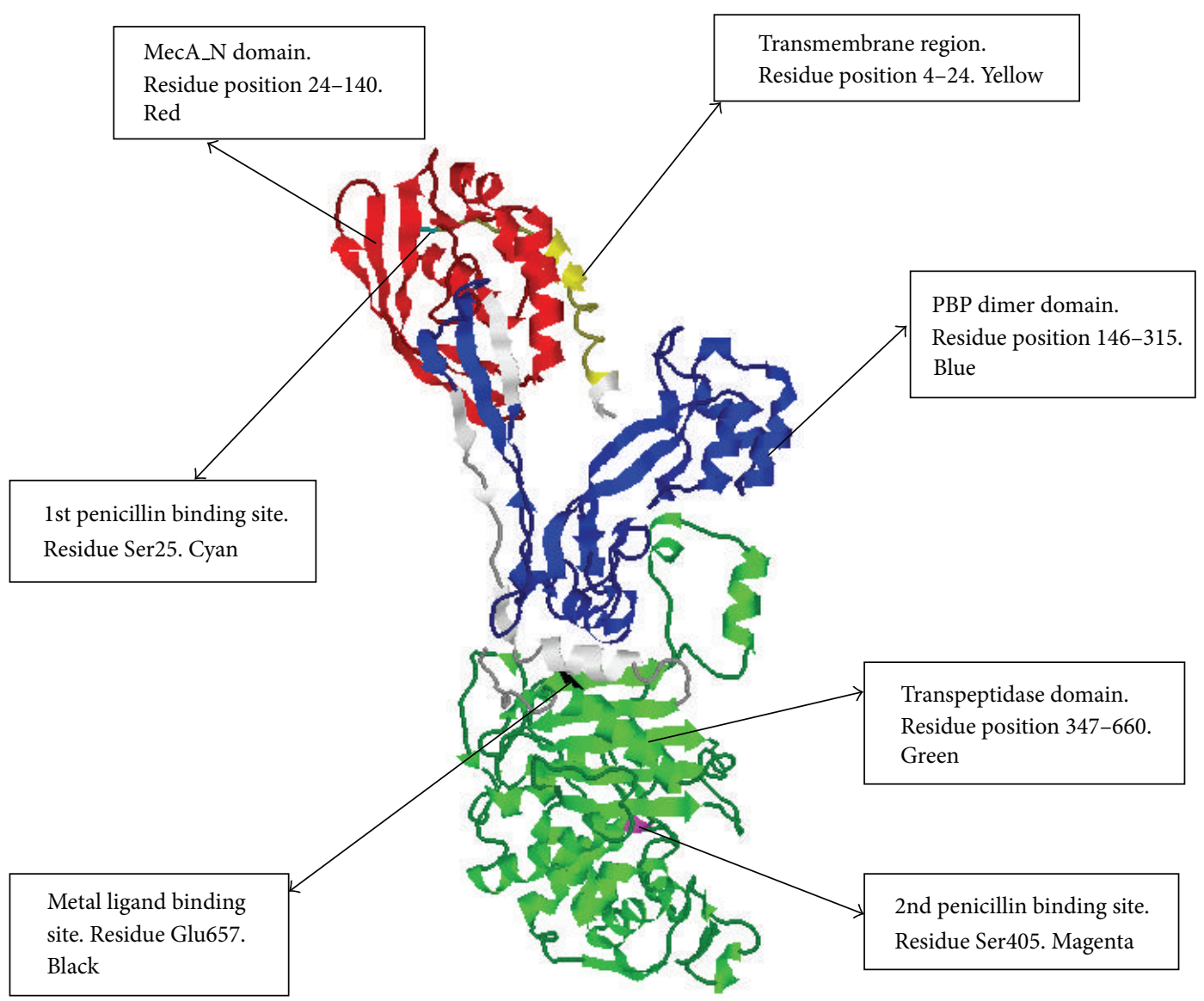

FIGURE 3: The functional domains distribution and position of regulatory amino acid residues on PBP- $2^{\prime}$ protein $3 \mathrm{D}$ structure.

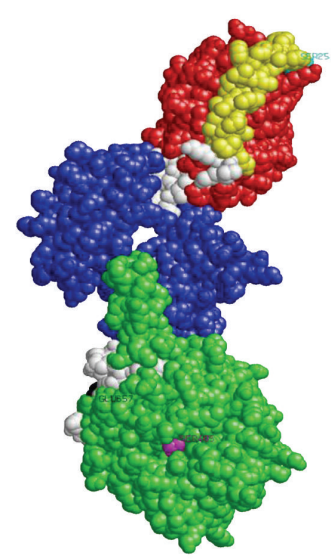

(a)

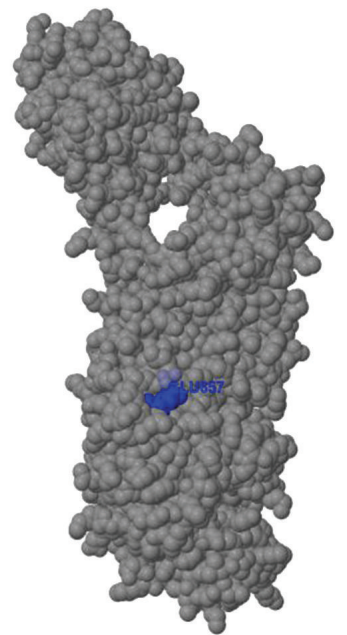

(b)

FIgURE 4: The position of the regulatory points and the binding of Glu657 residue to $\mathrm{Zn}^{2+}$ ion on PBP- $2^{\prime}$ protein folded structure. (a) On the folded structure the first penicillin binding site Ser25 (color cyan) and the metallic ligand binding site Glu657 (color black) are located on the surface while the second penicillin binding site Ser405 (color magenta) is not exposed on the surface but situated in an active site cavity. (b) This shows the $\mathrm{Zn}^{2+}$ ion (color grey) binding to the Glu657 residue (color blue) on the protein surface. 
B PBP with a TPase domain and a penicillin-insensitive second domain whose function is not known [71]. It is generally supported that membrane-bound transpeptidase which catalyzes the cross-linking of neighboring amino acid side chains during cell wall peptidoglycan synthesis is a major target for $\beta$ lactam action and that inhibition of transpeptidase enzyme leads to cell death $[72,73]$. Further, the transpeptidase domain is required for cell wall protein anchoring and virulence in MRSA [74]. The transpeptidase domain also contains two regulatory point for the PBP- $2^{\prime}$ gene. This includes the second penicillin binding site located at residue Ser405 (color magenta). The second penicillin binding site is also known as the active site or catalytic residue of the enzyme (Figures 3 and 4) [75].

As indicated above for Ser25 (first penicillin binding site), Ser405 might be regulated using amino acid substitutions combined with site-directed mutagenesis studies [62]. The Ser405 active site is very stable in the active fold linking with other residues through a network of hydrogen bonds. There is only one metallic ligand binding residue at Glu657 depicted black in color (Figures 3 and 4). It is exposed on the surface of the protein for easy binding to free metallic ligand such as $\mathrm{Zn}^{2+}$ ions (Figures 3 and 4).The Glu residue has been document as one of the essential residue sites involved when metals bind to proteins $[76,77]$, and Ser is also known as one of the key phosphorylation residues in proteins [78-80]. Metallic ions have been demonstrated as essential contributors to protein phorphorylation [81-85]. This suggests that in PBP- $2^{\prime}$ gene transpeptidase activity might involved or could be regulated by phorphorylation through the binding of metallic ions as ligands. Our analysis indicates that $\mathrm{Zn}^{2+}$ ions might be the key metallic ligand regulating the transpeptidase activity in MRSA. Polyoxotungstates in combination with beta lactam antibiotics make the MRSA strains more susceptible [86]. Also polyoxotungstates can be interchangeable substituted by transitional metal [87]. Therefore, the transitional metallic $\mathrm{Zn}^{2+}$ ions could be regulated by using appropriate ligands to improve the susceptibility of the MRSA to common $\beta$ lactam antibiotics. This finding is an important contribution in identifying new chemotherapeutic strategies for MRSA.

\section{Conclusions}

Previous studies and computer aided design drug screenings have been focusing mostly on penicillin binding protein $2 \mathrm{~A}$ (PBP2A) as the virulent factor of most of the MRSA associated infections [38]. Another key component of methicillinresistant mechanism is an acquired penicillin-binding protein (PBP), PBP-2', which has unusually low affinity for all $\beta$-lactam antibiotics and most other antibiotics [88]. We have elucidated the distinctive structural features of PBP-2' protein which can be exploited as chemotherapeutic targets for MSRA. The PBP-2' consist of 3 domains of MecA, PBP dimer and transpeptidase. The structural regulatory points consist of penicillin binding sites at Ser25, Ser405, and a metallic ligand binding site at Glu657. These findings offer new insight into the dynamic and functional determinants of PBP- $2^{\prime}$ in developing novel chemotherapeutic agents for MSRA.

\section{Conflict of Interests}

The authors report no conflict of interests in this work including the mentioned trademarks.

\section{Acknowledgments}

The authors would like to thank National Institutes of Health: Mississippi IDeA Network for Biomedical Research Excellence (NIH-NCRR-P20RR016476 and NIH-NIGMS8P20GM103476); Bioinformatics Programs in Minority Institutions (1T36GM095335); National Center for Integrative Biomedical Informatics (U54DA021519). They also would like to thank National Science Foundation: Mississippi NSFEPSCoR Grant Awards (EPS-0903787 and EPS 1006883); Undergraduate Research and Mentoring Program (DBI0958179) and Visual Analytics in Biology Curriculum Network (DBI-1062057). They also would like to acknowledge US Department of Homeland Security Science \& Technology Directorate (2011-ST-062-000048). The views and conclusions contained in this document are those of the authors and should not be interpreted as necessarily representing the official policies, either expressed or implied, of the funding agencies.

\section{References}

[1] K. Ubukata, N. Yamashita, and M. Konno, "Occurrence of a $\beta$-lactam-inducible penicillin-binding protein in methicillinresistant staphylococci," Antimicrobial Agents and Chemotherapy, vol. 27, no. 5, pp. 851-857, 1985.

[2] J. K. Collins, J. T. Mader, and M. T. Kelly, "Resistance of methicillin-resistant Staphylococcus aureus to third-generation cephalosporins," Journal of Infectious Diseases, vol. 147, no. 3, p. $591,1983$.

[3] C. F. Gravenkemper, J. L. Brodie, and W. M. Kirby, "Resisistance of coagulase positive Staphylococci to methicillin and oxacillin," Journal of Bacteriology, vol. 89, pp. 1005-1010, 1965.

[4] D. J. Diekema, M. A. Pfaller, F. J. Schmitz et al., "Survey of infections due to Staphylococcus species: frequency of occurrence and antimicrobial susceptibility of isolates collected in the United States, Canada, Latin America, Europe, and the Western Pacific region for the SENTRY Antimicrobial Surveillance Program, 1997-1999," Clinical Infectious Diseases, vol. 32, no. 10, supplement 2, pp. S114-S132, 2001.

[5] R. Geisel, F. Schmitz, A. Fluit, and H. Labischinski, "Emergence, mechanism, and clinical implications of reduced glycopeptide susceptibility in Staphylococcus aureus," European Journal of Clinical Microbiology and Infectious Diseases, vol. 20, no. 10, pp. 685-697, 2001.

[6] K. G. Dyke, "Penicillinase production and intrinsic resistance to penicillins in methicillin-resistant cultures of Staphylococcus aureus," Journal of Medical Microbiology, vol. 2, no. 3, pp. 261278, 1969.

[7] L. D. Sabath, S. J. Wallace, and D. A. Gerstein, "Suppression of intrinsic resistance to methicillin and other penicillins in 
Staphylococcus aureus," Antimicrobial Agents and Chemotherapy, vol. 2, no. 5, pp. 350-355, 1972.

[8] M. Laverdiere, D. Welter, and L. D. Sabath, "Use of a heavy inoculum in the in vitro evaluation of the antistaphylococcal activity of 19 cephalosporins," Antimicrobial Agents and Chemotherapy, vol. 13, no. 4, pp. 669-675, 1978.

[9] S. J. Seligman, "Penicillinase-negative variants of methicillinresistant Staphylococcus aureus," Nature, vol. 209, no. 5027, pp. 994-996, 1966.

[10] S. Boyle-Vavra, S. Yin, M. Challapalli, and R. S. Daum, “Transcriptional induction of the penicillin-binding protein 2 gene in Staphylococcus aureus by cell wall-active antibiotics oxacillin and vancomycin," Antimicrobial Agents and Chemotherapy, vol. 47, no. 3, pp. 1028-1036, 2003.

[11] B. G. Spratt, "Penicillin-binding proteins and the future of $\beta$ lactam antibiotics. The seventh Fleming lecture," Journal of General Microbiology, vol. 129, no. 5, pp. 1247-1260, 1983.

[12] N. H. Georgopapadakou, S. A. Smith, and D. P. Bonner, "Penicillin-binding proteins in a Staphylococcus aureus strain resistant to specific $\beta$-lactam antibiotics," Antimicrobial Agents and Chemotherapy, vol. 22, no. 1, pp. 172-175, 1982.

[13] B. Hartman and A. Tomasz, "Altered penicillin-binding proteins in methicillin-resistant strains of Staphylococcus aureus," Antimicrobial Agents and Chemotherapy, vol. 19, no. 5, pp. 726735, 1981.

[14] N. H. Georgopapadakou and F. Y. Liu, "Binding of $\beta$-lactam antibiotics to penicillin-binding proteins of Staphylococcus aureus and Streptococcus faecalis: relation to antibacterial activity," Antimicrobial Agents and Chemotherapy, vol. 18, no. 5, pp. 834-836, 1980.

[15] G. C. Stewart and E. D. Rosenblum, "Transduction of methicillin resistance in Staphylococcus aureus: recipient effectiveness and beta-lactamase production," Antimicrobial Agents and Chemotherapy, vol. 18, no. 3, pp. 424-432, 1980.

[16] H. F. Chambers, "Methicillin resistance in staphylococci: molecular and biochemical basis and clinical implications," Clinical Microbiology Reviews, vol. 10, no. 4, pp. 781-791, 1997.

[17] M. G. Pinho, H. De Lencastre, and A. Tomasz, "An acquired and a native penicillin-binding protein cooperate in building the cell wall of drug-resistant staphylococci," Proceedings of the National Academy of Sciences of the United States of America, vol. 98, no. 19, pp. 10886-10891, 2001.

[18] M. G. Pinho, A. M. Ludovice, S. Wu, and H. De Lencastre, "Massive reduction in methicillin resistance by transposon inactivation of the normal PBP2 in a methicillin-resistant strain of Staphylococcus aureus," Microbial Drug Resistance, vol. 3, no. 4, pp. 409-413, 1997.

[19] M. D. Song, M. Wachi, M. Doi, F. Ishino, and M. Matsuhashi, "Evolution of an inducible penicillin-target protein in methicillin-resistant Staphylococcus aureus by gene fusion," FEBS Letters, vol. 221, no. 1, pp. 167-171, 1987.

[20] T. Ito, Y. Katayama, K. Asada et al., "Structural comparison of three types of staphylococcal cassette chromosome mec integrated in the chromosome in methicillin-resistant Staphylococcus aureus," Antimicrobial Agents and Chemotherapy, vol. 45, pp. 1323-1336, 2001.

[21] Y. Katayama, T. Ito, and K. Hiramatsu, "A new class of genetic element, staphylococcus cassette chromosome mec, encodes methicillin resistance in Staphylococcus aureus," Antimicrobial Agents and Chemotherapy, vol. 44, no. 6, pp. 1549-1555, 2000.
[22] X. M. Xiao, T. Ito, C. Tiensasitorn et al., "Novel type of staphylococcal cassette chromosome mec identified in communityacquired methicillin-resistant Staphylococcus aureus strains," Antimicrobial Agents and Chemotherapy, vol. 46, no. 4, pp. 1147$1152,2002$.

[23] L. A. Kelley and M. J. E. Sternberg, "Protein structure prediction on the Web: a case study using the Phyre server," Nature protocols, vol. 4, no. 3, pp. 363-371, 2009.

[24] M. N. Wass, L. A. Kelley, and M. J. E. Sternberg, "3DLigandSite: predicting ligand-binding sites using similar structures," Nucleic Acids Research, vol. 38, no. 2, Article ID gkq406, pp. W469W473, 2010.

[25] I. N. Berezovsky and E. N. Trifonov, "Van der Waals locks: loop-n-lock structure of globular proteins," Journal of Molecular Biology, vol. 307, no. 5, pp. 1419-1426, 2001.

[26] Y. W. Chen, A. R. Fersht, and K. Henrick, "Contribution of buried hydrogen bonds to protein stability. The crystal structures of two barnase mutants," Journal of Molecular Biology, vol. 234, no. 4, pp. 1158-1170, 1993.

[27] A. Sillero and A. Maldonado, "Isoelectric point determination of proteins and other macromolecules: oscillating method," Computers in Biology and Medicine, vol. 36, no. 2, pp. 157-166, 2006.

[28] C. M. Stoscheck, "Quantitation of protein," Methods in Enzymology, vol. 182, pp. 50-68, 1990.

[29] S. C. Gill and P. H. Von Hippel, "Calculation of protein extinction coefficients from amino acid sequence data," Analytical Biochemistry, vol. 182, no. 2, pp. 319-326, 1989.

[30] A. Bachmair, D. Finley, and A. Varshavsky, "In vivo half-life of a protein is a function of its amino-terminal residue," Science, vol. 234, no. 4773, pp. 179-186, 1986.

[31] D. K. Gonda, A. Bachmair, I. Wunning, J. W. Tobias, W. S. Lane, and A. Varshavsky, "Universality and stucture of the Nend rule," Journal of Biological Chemistry, vol. 264, no. 28, pp. 16700-16712, 1989.

[32] A. Varshavsky, "The N-end rule pathway of protein degradation," Genes to Cells, vol. 2, no. 1, pp. 13-28, 1997.

[33] K. Guruprasad, B. V. B. Reddy, and M. W. Pandit, "Correlation between stability of a protein and its dipeptide composition: a novel approach for predicting in vivo stability of a protein from its primary sequence," Protein Engineering, vol. 4, no. 2, pp. 155$161,1990$.

[34] A. Ikai, "Thermostability and aliphatic index of globular proteins," Journal of Biochemistry, vol. 88, no. 6, pp. 1895-1898, 1980.

[35] J. Kyte and R. F. Doolittle, "A simple method for displaying the hydropathic character of a protein," Journal of Molecular Biology, vol. 157, no. 1, pp. 105-132, 1982.

[36] T. P. Hopp and K. R. Woods, "Prediction of protein antigenic determinants from amino acid sequences," Proceedings of the National Academy of Sciences of the United States of America, vol. 78, no. 6, pp. 3824-3828, 1981.

[37] H. F. Chambers and F. R. DeLeo, "Waves of resistance: Staphylococcus aureus in the antibiotic era," Nature Reviews Microbiology, vol. 7, no. 9, pp. 629-641, 2009.

[38] S. Skariyachan, R. S. Krishnan, S. B. Siddapa, C. Salian, P. Bora, and D. Sebastian, "Computer aided screening and evaluation of herbal therapeutics against MRSA infections," Bioinformation, vol. 7, no. 5, pp. 222-233, 2011.

[39] D. C. Oliveira and H. de Lencastre, "Methicillin-resistance in Staphylococcus aureus is not affected by the overexpression in trans of the mecA gene repressor: a surprising observation," PLoS ONE, vol. 6, no. 8, Article ID e23287, 2011. 
[40] B. D. Wines, H. M. Trist, W. Farrugia et al., "A conserved host and pathogen recognition site on immunoglobulins: structural and functional aspects," Advances in Experimental Medicine and Biology, vol. 946, pp. 87-112, 2012.

[41] S. B. Pandit and N. Srinivasan, "Survey for G-proteins in the prokaryotic genomes: prediction of functional roles based on classification," Proteins, vol. 52, no. 4, pp. 585-597, 2003.

[42] J. Colicelli, "Human RAS superfamily proteins and related GTPases," Science's STKE, vol. 2004, no. 250, p. RE13, 2004.

[43] L. Helft, V. Reddy, X. Chen et al., "LRR conservation mapping to predict functional sites within protein leucine-rich repeat domains," PLoS ONE, vol. 6, no. 7, Article ID e21614, 2011.

[44] W. D. Beck, B. Berger-Bachi, and F. H. Kayser, "Additional DNA in methicillin-resistant Staphylococcus aureus and molecular cloning of mec-specific DNA," Journal of Bacteriology, vol. 165, no. 2, pp. 373-378, 1986.

[45] T. Ito, Y. Katayama, and K. Hiramatsu, "Cloning and nucleotide sequence determination of the entire mec DNA of premethicillin-resistant Staphylococcus aureus N315," Antimicrobial Agents and Chemotherapy, vol. 43, no. 6, pp. 1449-1458, 1999.

[46] K. Hiramatsu, K. Asada, E. Suzuki, K. Okonogi, and T. Yokota, "Molecular cloning and nucleotide sequence determination of the regulator region of mecA gene in methicillin-resistant Staphylococcus aureus (MRSA)," FEBS Letters, vol. 298, no. 2-3, pp. 133-136, 1992.

[47] K. Kuwahara-Arai, N. Kondo, S. Hori, E. Tateda-Suzuki, and K. Hiramatsu, "Suppression of methicillin resistance in a mecA-containing pre- methicillin-resistant Staphylococcus aureus strain is caused by the mecI-mediated repression of PBP $2^{\prime}$ production," Antimicrobial Agents and Chemotherapy, vol. 40, no. 12 , pp. 2680-2685, 1996.

[48] T. M. Dickinson and G. L. Archer, "Phenotypic expression of oxacillin resistance in Staphylococcus epidermidis: roles of mecA transcriptional regulation and resistant-subpopulation selection," Antimicrobial Agents and Chemotherapy, vol. 44, no. 6, pp. 1616-1623, 2000.

[49] G. L. Archer, D. M. Niemeyer, J. A. Thanassi, and M. J. Pucci, "Dissemination among staphylococci of DNA sequences associated with methicillin resistance," Antimicrobial Agents and Chemotherapy, vol. 38, no. 3, pp. 447-454, 1994.

[50] R. L. Hurlimann-Dalel, C. Ryffel, F. H. Kayser, and B. Berger-Bachi, "Survey of the methicillin resistance-associated genes mecA, mecRl-mecI, and femA-femB in clinical isolates of methicillin-resistant Staphylococcus aureus," Antimicrobial Agents and Chemotherapy, vol. 36, no. 12, pp. 2617-2621, 1992.

[51] N. Kobayashi, K. Taniguchi, and S. Urasawa, "Analysis of diversity of mutations in the mecI gene and mecA promoter/operator region of methicillin-resistant Staphylococcus aureus and Staphylococcus epidermidis," Antimicrobial Agents and Chemotherapy, vol. 42, no. 3, pp. 717-720, 1998.

[52] E. Suzuki, K. Kuwahara-Arai, J. F. Richardson, and K. Hiramatsu, "Distribution of mec regulator genes in methicillinresistant Staphylococcus clinical strains," Antimicrobial Agents and Chemotherapy, vol. 37, no. 6, pp. 1219-1226, 1993.

[53] T. M. A. Weller, "The distribution of mecA, mecR1 and mecI and sequence analysis of mecI and the mec promoter region in staphylococci expressing resistance to methicillin," Journal of Antimicrobial Chemotherapy, vol. 43, no. 1, pp. 15-22, 1999.

[54] B. J. Hartman and A. Tomasz, "Expression of methicillin resistance in heterogeneous strains of Staphylococcus aureus," Antimicrobial Agents and Chemotherapy, vol. 29, no. 1, pp. 8592, 1986.
[55] K. Murakami and A. Tomasz, "Involvement of multiple genetic determinants in high-level methicillin resistance in Staphylococcus aureus," Journal of Bacteriology, vol. 171, no. 2, pp. 874879, 1989.

[56] D. C. Oliveira, A. Tomasz, and H. De Lencastre, "The evolution of pandemic clones of methicillin-resistant Staphylococcus aureus: identification of two ancestral genetic backgrounds and the associated mec elements," Microbial Drug Resistance, vol. 7, no. 4, pp. 349-361, 2001.

[57] M. C. Enright, D. A. Robinson, G. Randle, E. J. Feil, H. Grundmann, and B. G. Spratt, "The evolutionary history of methicillin-resistant Staphylococcus aureus (MRSA)," Proceedings of the National Academy of Sciences of the United States of America, vol. 99, no. 11, pp. 7687-7692, 2002.

[58] C. Kim, C. Milheiriço, S. Gardete et al., "Properties of a novel PBP2A protein homolog from Staphylococcus aureus strain LGA251 and its contribution to the $\beta$-lactam-resistant phenotype," Journal of Biological Chemistry, vol. 287, no. 44, pp. 36854-36863, 2012.

[59] N. Matsuda, H. Hayashi, S. Miyatake, T. Kuroiwa, and H. Kagamiyama, "Instability of the apo form of aromatic L-amino acid decarboxylase in vivo and in vitro: implications for the involvement of the flexible loop that covers the active site," Journal of Biochemistry, vol. 135, no. 1, pp. 33-42, 2004.

[60] A. Subramani and C. A. Floudas, "Structure prediction of loops with fixed and flexible stems," Journal of Biological Chemistry, vol. 116, no. 23, pp. 6670-6682, 2012.

[61] A. Zervosen, R. Herman, F. Kerff et al., "Unexpected tricovalent binding mode of boronic acids within the active site of a penicillin-binding protein," Journal of the American Chemical Society, vol. 133, no. 28, pp. 10839-10848, 2011.

[62] N. N. Qureshi, D. Morikis, and N. L. Schiller, "Contribution of specific amino acid changes in penicillin binding protein 1 to amoxicillin resistance in clinical Helicobacter pylori isolates," Antimicrobial Agents and Chemotherapy, vol. 55, no. 1, pp. 101109, 2011.

[63] C. A. L. Zijderveld, M. E. G. Aarsman, T. Den Blaauwen, and N. Nanninga, "Penicillin-binding protein 1B of Escherichia coli exists in dimeric forms," Journal of Bacteriology, vol. 173, no. 18, pp. 5740-5746, 1991.

[64] T. Den Blaauwen, F. B. Wientjes, A. H. J. Kolk, B. G. Spratt, and N. Nanninga, "Preparation and characterization of monoclonal antibodies against native membrane-bound penicillin-binding protein 1B of Escherichia coli," Journal of Bacteriology, vol. 171, no. 3, pp. 1394-1401, 1989.

[65] A. Edelman, L. Bowler, J. K. Broome-Smith, and B. G. Spratt, "Use of a beta-lactamase fusion vector to investigate the organization of penicillin-binding protein $1 \mathrm{~B}$ in the cytoplasmic membrane of Escherichia coli," Molecular Microbiology, vol. 1, no. 1, pp. 101-106, 1987.

[66] A. Rodriguez-Tebar, J. A. Barbas, and D. Vazquez, "Location of some proteins involved in peptidoglycan synthesis and cell division in the inner and outer membranes of Escherichia coli," Journal of Bacteriology, vol. 161, no. 1, pp. 243-248, 1985.

[67] C. Chalut, M.-H. Remy, and J.-M. Masson, "Disulfide bridges are not involved in penicillin-binding protein $1 \mathrm{~b}$ dimerization in Escherichia coli," Journal of Bacteriology, vol. 181, no. 9, pp. 2970-2972, 1999.

[68] J.-V. Höltje, "Growth of the stress-bearing and shapemaintaining murein sacculus of Escherichia coli," Microbiology and Molecular Biology Reviews, vol. 62, no. 1, pp. 181-203, 1998. 
[69] N. Nanninga, "Morphogenesis of Escherichia coli," Microbiology and Molecular Biology Reviews, vol. 62, no. 1, pp. 110-129, 1998.

[70] M. G. Pinho, S. R. Filipe, H. De Lencastre, and A. Tomasz, "Complementation of the essential peptidoglycan transpeptidase function of penicillin-binding protein 2 (PBP2) by the drug resistance protein PBP2A in Staphylococcus aureus," Journal of Bacteriology, vol. 183, no. 22, pp. 6525-6531, 2001.

[71] C. Goffin and J.-M. Ghuysen, "Multimodular penicillin-binding proteins: an enigmatic family of orthologs and paralogs," Microbiology and Molecular Biology Reviews, vol. 62, no. 4, pp. 10791093, 1998.

[72] D. J. Tipper and J. L. Strominger, "Mechanism of action of penicillins: a proposal based on their structural similarity to acyl-D-alanyl-D-alanine," Proceedings of the National Academy of Sciences of the United States of America, vol. 54, no. 4, pp. 11331141, 1965.

[73] E. M. Wise Jr. and J. T. Park, "Penicillin: its basic site of action as an inhibitor of a peptide cross-linking reaction in cell wall mucopeptide synthesis," Proceedings of the National Academy of Sciences of the United States of America, vol. 54, no. 1, pp. 75-81, 1965.

[74] B. A. Frankel, M. Bentley, R. G. Kruger, and D. G. McCafferty, "Vinyl sulfones: inhibitors of SrtA, a transpeptidase required for cell wall protein anchoring and virulence in Staphylococcus aureus," Journal of the American Chemical Society, vol. 126, no. 11, pp. 3404-3405, 2004.

[75] R. Wu, S. Richter, R.-G. Zhang, V. J. Anderson, D. Missiakas, and A. Joachimiak, "Crystal structure of Bacillus anthracis transpeptidase enzyme CapD," Journal of Biological Chemistry, vol. 284, no. 36, pp. 24406-24414, 2009.

[76] A. Passerini, M. Punta, A. Ceroni, B. Rost, and P. Frasconi, "Identifying cysteines and histidines in transition-metalbinding sites using support vector machines and neural networks," Proteins, vol. 65, no. 2, pp. 305-316, 2006.

[77] M. M. Harding, "The architecture of metal coordination groups in proteins," Acta Crystallographica D, vol. 60, no. 5, pp. 849859, 2004.

[78] T. Suzuki, K. Kita, and T. Ochi, "Phosphorylation of histone H3 at serine 10 has an essential role in arsenite-induced expression of FOS, EGR1 and IL8 mRNA in cultured human cell lines," Journal of Applied Toxicology, vol. 33, no. 8, pp. 746-755, 2013.

[79] S.-I. Hu, M. Katz, S. Chin et al., "MNK2 inhibits eIF4G activation through a pathway involving serine-arginine-rich protein kinase in skeletal muscle," Science Signaling, vol. 5, no. 211, article ral4, 2012.

[80] K. Kettner, U. Krause, S. Mosler, C. Bodenstein, T. M. Kriegel, and G. Rödel, "Saccharomyces cerevisiae gene YMR291W/TDA1 mediates the in vivo phosphorylation of hexokinase isoenzyme 2 at serine-15," FEBS Letters, vol. 586, no. 4, pp. 455-458, 2012.

[81] A. N. Mbah, O. Mahmud, O. R. Awofolu, and R. D. Isokpehi, "Inferences on the biochemical and environmental regulation of universal stress proteins from Schistosomiasis parasites," Advances and Applications in Bioinformatics and Chemistry, vol. 6, pp. 15-27, 2013.

[82] A. N. Mbah, H. Kamga, O. R. Awofolu, and R. D. Isokpehi, "Drug target exploitable structural features of adenylyl cyclase activity in Schistosoma mansoni," Drug Target Insights, vol. 6, pp. 41-58, 2012.

[83] R. Luo, C. Zhou, J. Lin, D. Yang, Y. Shi, and G. Cheng, "Identification of in vivo protein phosphorylation sites in human pathogen Schistosoma japonicum by a phosphoproteomic approach," Journal of Proteomics, vol. 75, no. 3, pp. 868$877,2012$.

[84] T. Nebl, J. H. Prieto, E. Kapp et al., "Quantitative in vivo analyses reveal calcium-dependent phosphorylation sites and identifies a novel component of the toxoplasma invasion motor complex," PLoS Pathogens, vol. 7, no. 9, Article ID e1002222, 2011.

[85] I. Kim, E. J. Park, J. Seo, S. J. Ko, J. Lee, and C. H. Kim, "Zinc stimulates tau S214 phosphorylation by the activation of Raf/mitogen-activated protein kinase-kinase/extracellular signal-regulated kinase pathway," NeuroReport, vol. 22, no. 16, pp. 839-844, 2011.

[86] T. Yamase, N. Fukuda, and Y. Tajima, "Synergistic effect of polyoxotungstates in combination with $\beta$-lactam antibiotics on antibacterial activity against methicillin-resistant Staphylococcus aureus," Biological and Pharmaceutical Bulletin, vol. 19, no. 3, pp. 459-465, 1996.

[87] J. A. F. Gamelas, A. R. Gaspar, D. V. Evtuguin, and C. Pascoal Neto, "Transition metal substituted polyoxotungstates for the oxygen delignification of kraft pulp," Applied Catalysis A, vol. 295, no. 2, pp. 134-141, 2005.

[88] J. T. Murphy, R. Walshe, and M. Devocelle, "A computational model of antibiotic-resistance mechanisms in methicillinresistant Staphylococcus aureus (MRSA)," Journal of Theoretical Biology, vol. 254, no. 2, pp. 284-293, 2008. 


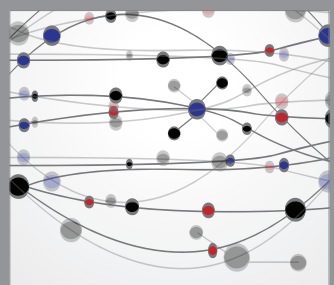

The Scientific World Journal
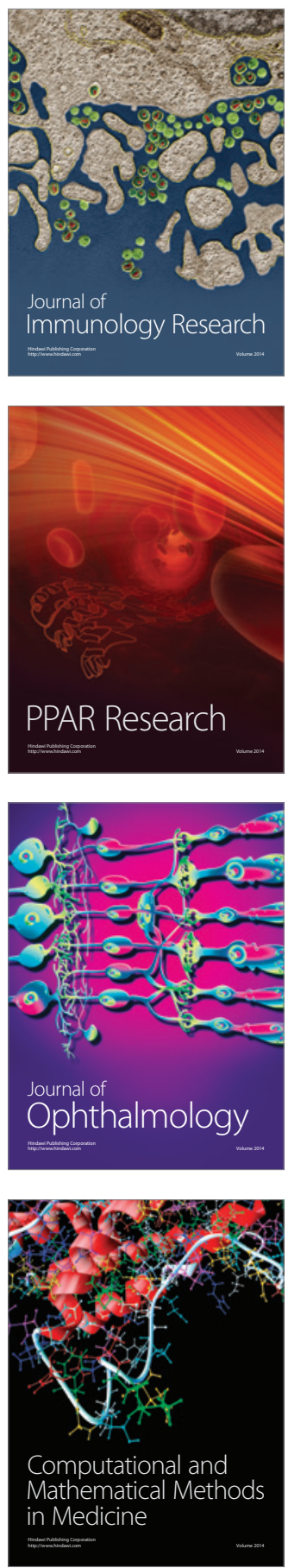

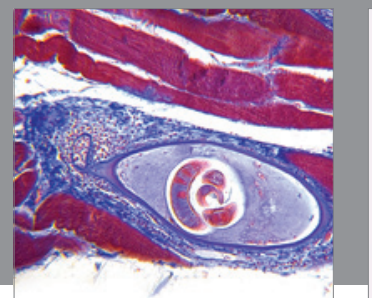

Gastroenterology

Research and Practice
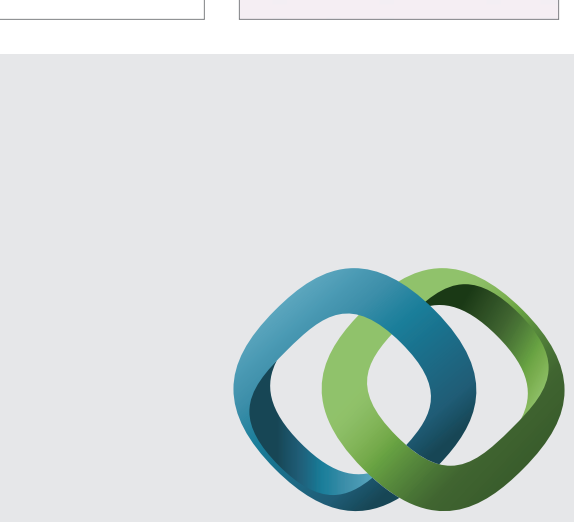

\section{Hindawi}

Submit your manuscripts at

http://www.hindawi.com
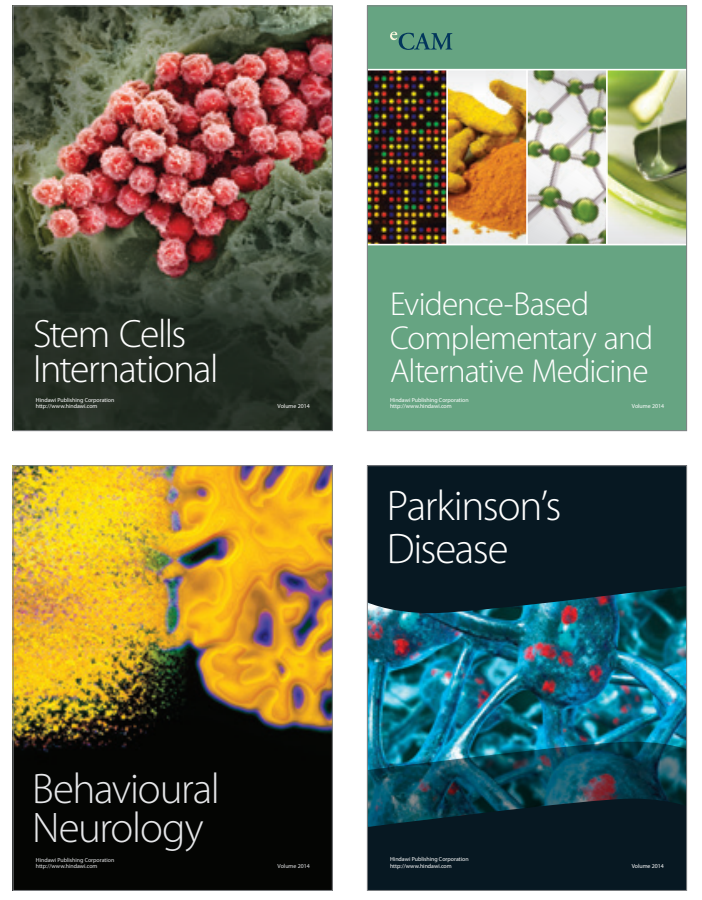
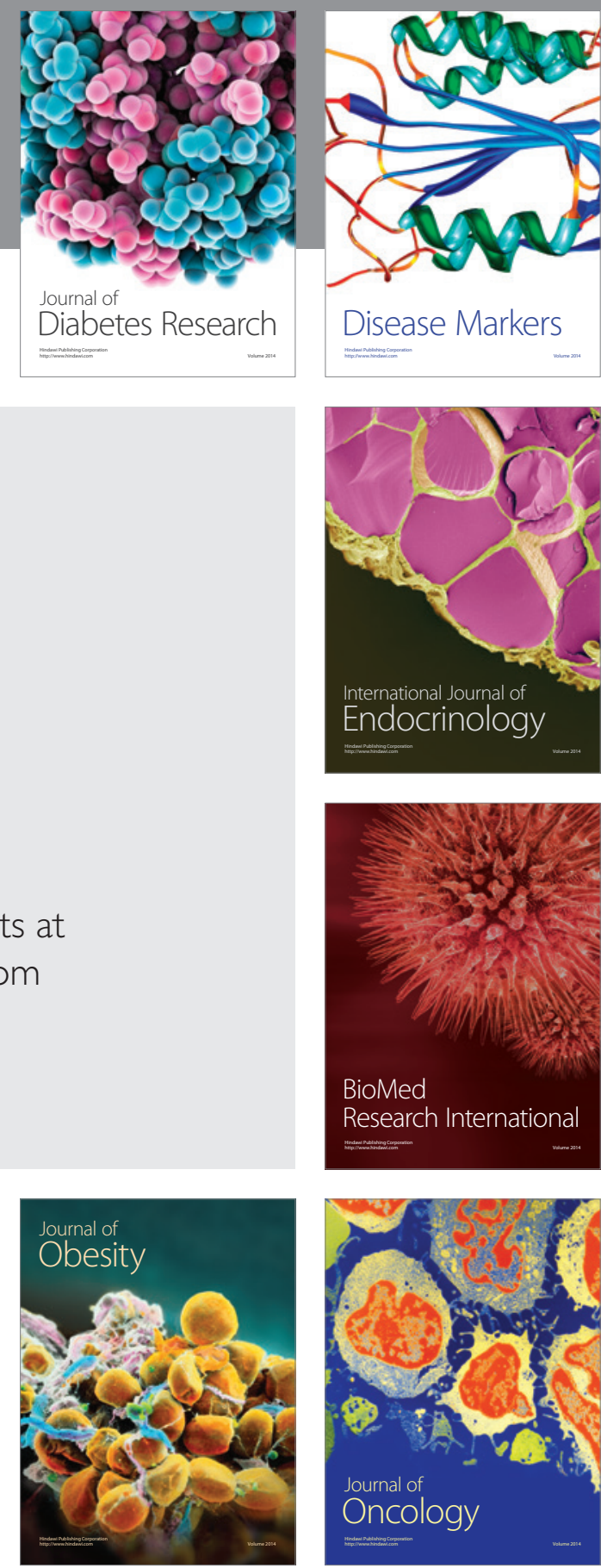

Disease Markers
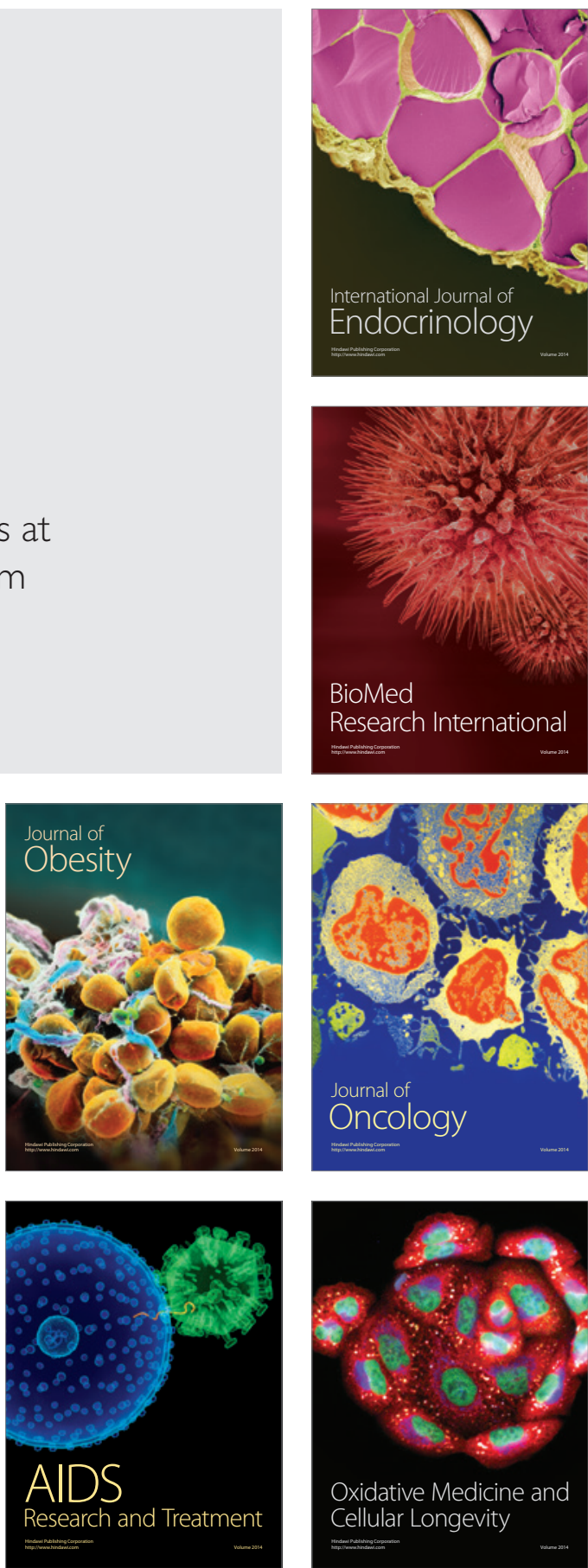